\title{
PATIENT WITH ANCA-ASSOCIATED VASCULITIS AND HYPEREOSINOPHILIA PRESENTING PULMONARY-KIDNEY SYNDROME
}

Vanessa Burini Lobo,, *, Fernanda Freire Kosac', Tiere Costa Bravim¹, Isabela Taveira Mouzinho', Luciana Nunes de Assis Daameche', Jamille Nascimento Carneiro', Carlos Eduardo de Carvalho Lins' ${ }^{1}$

1. Hospital de Base do Distrito Federal, Brasília (DF), Brazil.

*Corresponding author: vanessalobo239@hotmail.com

\section{BACKGROUND}

Pulmonary-renal syndrome (PRS) is defined as the coexistence of diffuse alveolar hemorrhage and glomerulonephritis. The following case presents a patient with PRS with positive P-ANCA, but with eosinophilia, suggestive of eosinophilic granulomatosis with polyangiitis (EGPA), but with no history of upper and lower airway involvement due to asthma or allergic rhinitis.

\section{CASE REPORT}

Male patient, 54 years old, with no previous comorbidities, with a history of asthenia, diarrhea without the presence of blood or mucus associated with unverified fever that started 1 month before hospital admission. After 15 days of symptom onset, he presented dyspnea, dry cough without hemoptoics episodes, symmetrical lower limb edema, hypertension, with laboratory tests showing acute kidney injury, $6.94 \mathrm{mg} / \mathrm{dL}$ creatinine, proliferative EAS, anemia and eosinophilia $\left(3360 \mathrm{cls} / \mathrm{mm}^{3}\right)$. The patient denied weight loss, dysuria, odynophagia, skin lesions, arthralgia, allergic rhinitis, asthma, alcoholism, smoking or use of illicit drugs. After 2 days of hospitalization, the patient evolved with the need for renal replacement therapy, and on the 4th day after admission, he needed invasive mechanical ventilation, due to acute respiratory failure, with bronchoscopy performed in the first 24 hours after orotracheal intubation with evidence of lavage serial bronchoalveolar hemorrhagic aspect, compatible with alveolar hemorrhage. He had P-ANCA titer 1:80, PCR: 23 mg/dL, antibasement membrane antibody, ANA, anti-DNA, C-ANCA, anti-PR3 negative, normal C3 and C4 and persistent eosinophilia. All viral, bacterial and parasitological investigations to assess infectious etiologies were negative. Renal biopsy showed crescent glomerulonephritis, type II pauci-immune form, with interstitial tubule space moderately enlarged by granulomonuclear infiltrate with plasmocytes and eosinophils (Figs. 1and 2). Pulse therapy with methylprednisolone $1 \mathrm{~g}$ for 3 days, 5 sessions of plasmapheresis, cyclophosphamide $1 \mathrm{~g}$, with resolution of alveolar hemorrhage, but the patient remains undergoing hemodialysis after 6 months of diagnosis.

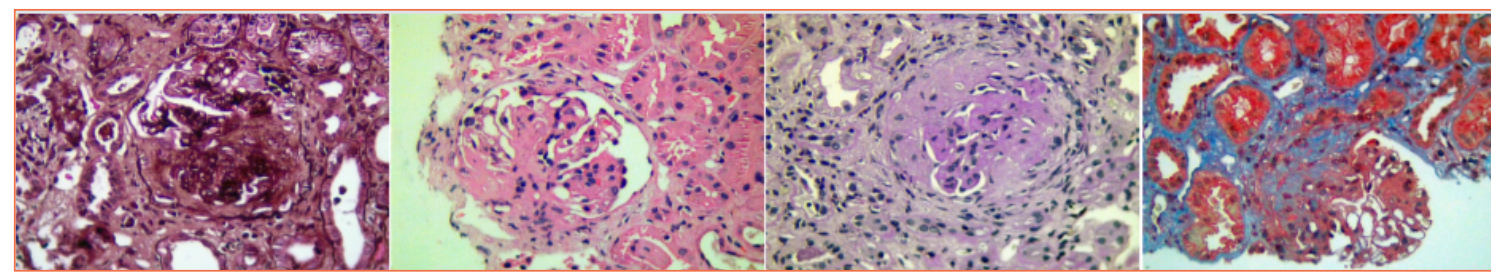

Figure 1. Glomerular compartment.

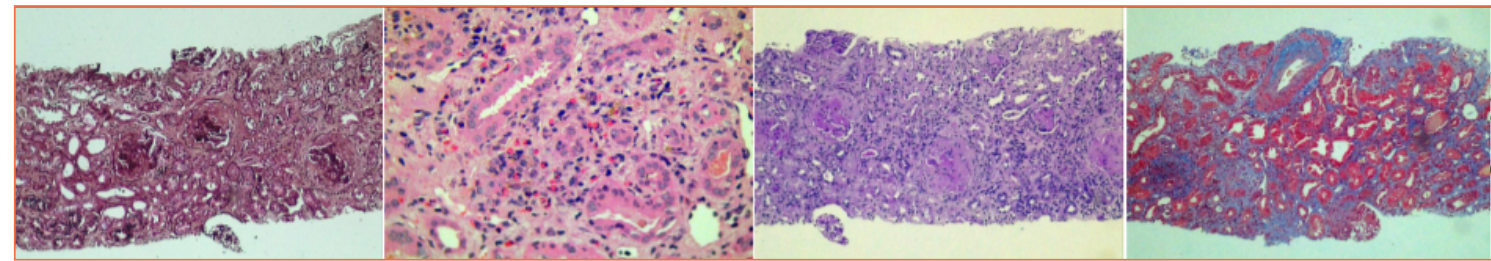

Figure 2. Tubulointerstitial compartment. 


\section{CONCLUSION}

This case has an atypical clinical presentation, both for not following the usual clinical course of the disease that occurs in three phases: rhinitis and asthma, then tissue eosinophilia and, finally, systemic vasculitis, and for not meeting the classification criteria by ACR 1990 for EGPA. However, renal biopsy was essential for the diagnosis, since pauci-immune necrotizing GN is characteristic of ANCA-associated vasculitides, but the finding of interstitial infiltrates with abundant eosinophils and plasma cells is typical of EGPA; the interstitium in other ANCA-related vasculitis is moderately populated by $T$ and $B$ lymphocytes. 